W. to $42^{\circ}{ }^{\prime} \mathrm{O}^{\prime} \mathrm{W}$. It certainly must appear singular to geographers that the limits of the best-known stream in the world should be so ill-defined; but the temperature of the sea at the places marked in the chart cannot suddenly change $12^{\circ}$ from any other cause than the irruption of the Gulf-stream or the ordinary waters of the ocean. Had it occurred in a single season only, the correctness of the observations might have been impugned; but extending, as they do, over several years, their accuracy cannot be challenged.

It is the opinion of many that the Gulf-stream is extending its boundaries northward, and ameliorating the climate of the British islands. Such an assumption is not an impossibility, although there are no changes of volume or velocity at its outlet into the Atlantic. There are, however, grounds for believing that the Labrador current does not run with its former force, as icebergs are seldom seen south of the parallel of $4.3^{\circ} 30^{\prime}$ north latitude. Observation can alone confirm this theory, but whether correct or not it in nowise affects the accuracy of my data.

WM. W. KIDDLE

U.S. White Star Mail Steamship Oceanic, Feb. 2

[We have received a chart from $\mathrm{Mr}$. Kiddle; but it is too large for insertion in NATURE.]

\section{A Lecture Experiment}

Mr. TAIT's letter in NATURE of February 26 calls to mincl an effective lecture illustration I have used in my classes to illus. trate a fog or cloud produced by cooling air containing moisture. Instead of using an air-pump as described in "Heat, a mode of Motion," take a flask of one or two litres capacity, rinse it out with distilled water, and attach to the neck a cork and glass tube of about twenty or thirty centimetres in Iength. Place the glass tube in the mouth and exhaust, when a dense cloud will be formed; then on blowing into the flask the cloud disappears. The cloud may be produced and dissclved as often as wished, and if a beam from the oxy-hydrogen light be sent through the flask, the experiment becomes very effective.

Midland Institute, Birmingham

C. J. WOODWARD

\section{The "Treasury of Botany"}

I'T might be inferred from your notice of the new edition of the "Treasury of Botany" (NATURE, vol. ix. p. 300) that the stereotyped pages of the original text of that work-of which you are pleased to speak in terms of commendation-had been reprinted withont alteration. Will you allow me space to state that this is by no means the case (as indeed is stated in the pretace), but that a large number of corrections have been made, as may be detected by a keen eye in consequence of the slight difference which is observable in the type where the alteration has extended over two or three lines or more. Hence it is not to the Supplement alone that the reader must look for such of the "additions to boinical knowledge made during the last eight years " as it has been found practicable to include in the revised edition.

Thos. MOOR

[WE are glad of the opportunity afforded by the foregoing letter of repeating our opinion, already expressed, that in the department of botanical nomenclature and classification, the new edition of the "Treasury of Botany" is an altogether admirabie and indispensable work. It is in this deparment only, or almost exclusively, that the corrections alluded to by Mr. Moore-and to which we perhaps ought to have called special attention-have been made, at least as far as we have been able to detect. We regret that we cannot withdraw from our statement that the same care has not been taken with the histological and physiological section. We might quote a number of instances in support of this assertion-a very ungracious task in speaking of a work so excellent in other respects - but will only refer to a single one. Notwithstanding that a very good and useful epitome of the more important properties of "Cellulose" is given in the Supplement, the statement is allowed to stand in the article in the bocly of the work, that "its composition, according to the latest analysie, is $\mathrm{C}_{\overline{2} 4} \mathrm{H}_{20} \mathrm{O}_{10}$ " a formula which does not, and never did, even under the old notation, represent anything near its composition, -A. W. B.]

\section{The Moons of Uranus}

IN your "Notes," this week, it is stated that since Mr. Lassell's observations at Malta, no one has seen the four moons of Uranus, until the re-discovery of the two small ones lately with the new Washington telescope.

In 1869-70, the planet was observed with the Melbourne reflector; the observations were specially directed to the disc, but at the same time the positions of the four satellites were noted on successive nights and thus identified,

I speak from memory, but have no doubt that the observations are to be found in the Mellourne records. The statement " have actually been measured by Prof. Newcomb, " probably refers to position, angle, and distance.

March I

L. S.

\section{MEN OF SCIENCE, THEIR NATURE AND THEIR NURTURE*}

$\mathrm{THE}$ lecturer spoke of the qualities by which the English men of science of the present day were characterised; he showed the possibility of defining and measuring the amount of any of those qualities, and concluded by summarising the opinions of the scientific men on the merits and demerits of their own education, and gave his interpretation of what, according to their own showing, they would have preferred. His data were obtained from a large collection of autobiographical notes, most obligingly communicated to him, in response to his requests, from the larger part of the leading members of the scientific world. He had addressed I 80 , who, being Fellows of the Royal Society, had, in addition, gained medals or filled posts of recognised scientific position; II 5 answers had already been received, of which so or yo were full and minute replies to his long and varied series of questions. He dealt with only a small part of his deductions from this valuable material, referring to a forthcoming work for the rest.

Regarding the chief qualities in the order of their prevalence among the scientific men, they were-(I) Energy both of body and mind; (2) Good health ; (3) Great independence of character ; (4) Tenacity of purpose ; (5) Practical business habits; and (6) What was usually the salt of the whole, strong innate tastes for science generally or some branch of it. He illustrated his remarks by reading many anonymous extracts from the returns, and explained in what way a notable deficiency in any of the above-mentioned qualities would tend to disqualify a man from succeeding in science.

As to the measurement of qualities, it was argued that the law of constancy in vital statistics might be taken for granted, being evidenced by the experience of insurance offices against fire, death, shipwreck, and other contingencies, always with the proviso that the facts are gathered with discretion, on well-known general principles. Hence we may say with assurance, that although two common nuts may differ, yet the contents of different packets, each containing $I, 000$ nuts, will be scarcely distinguishable, for the same number of nuts of different sizes will be found in each. Let the contents of the several packets be each arranged in a long row, in order of size, beginning with the biggest nut and ending with the smallest, and place the rows rank behind rank; then by the law of statistical constancy the nuts in the same files will in all cases be closely alike (except the outside ones, where more irregularity prevails). Again, if we incorporate two rows into one of double length, still preserving the arrangement as to regular gradation in size, the centre nuts of the two original series will still be found at or near the centre of the compound series, the nuts in quarter positions will still be in quarter positions, and so on. Hence, whatever be the length of the series the relative position in it of the nut will be a strict criterion of its size. This is of course equally true of all groups of qualities or characters

* Lecture on Friday evening, Feb. 27 , at the Royal Institution, by Franci Galton, F.R.S. 
whatever, in which the law of statistical constancy prevails, the series, in each case, being arranged according to gradations of the quality in question. Each individual is measured against his neighbour, and it is quite unnecessary to have recourse to any external standard. As regards a scale of equal parts, the lecturer made use of a converse application of the law of "frequency of error," which he illustrated by many experiments, and which showed that in a row (say as before) of nuts, if we took those which occupied the three quarterly divisions (rst quarter, centre, 3rả quarter) as the three elementary graduations of size, a range of successive graduations would be obtained by the following series, in which the places of the nuts are supposed to be reckoned from the end of the row where the large nuts are situated, and to be given in per-thousandths of the entire length of the row. It might be called the "Common Statistical Scale" (S. S.). The place of $+4^{\circ}$ would be at 4 thousandths from large end; $+3^{\circ}$, at 21 thousandths; $+2^{\circ}$ at $89 ;+1$, at $250 ; 0^{\circ}$ at $500 ;-1^{\circ}$ at $750 ;-2^{\circ}$ at $911 ;-3^{\circ}$ at 979 ; and $-4^{\circ}$ at 996 , or 4 thousandths from the small end of the row. Thus if we say that the size of a nut is $+2^{\circ} \mathrm{S}$. S., we absolutely define it. Anybody can procure such a nut independently by getting a quart of nuts and arranging them. Also we know that the difference between a nut of $+4^{\circ}$ S. S. and $+\mathrm{I}^{\circ}$ S.S. is $3^{\circ}$, and therefore three times as great as between one of $+2^{\circ} \mathrm{S}$. S. and the latter. It cannot be affirmed that this is a precise scale of equal parts for all qualities, but it is found to hold surprisingly well in a great variety of vital statistics; perhaps, too, the mere thickness of tissues may be a chief element in the physical basis of life. This scale appears, at all events, more likely to be nearly approximative to one of equal parts, for qualities generally, than any other that can be specified, and it certainly affords definite standards subject to the law of statistical constancy. The habit should therefore be encouraged in biographies, of giving copious illustrations which tend to rank a man among his contemporaries, in respect to every quality that is discussed, in order to give data for appraising those qualities in terms of the Statistical Scale. By the general use of a system of measurement like the above, social and political science would be greatly raised in precision.

Regarding education, the lecturer disavowed speaking of what might be suitable for boys generally, but he summarised the replies of the scientific men with reference to their own special experience, and notwithstanding the diversity of branches of science, he found unanimity in their replies. They commonly expressed a hatred of grammar and classics, the old-fashioned system of education being utterly distasteful to them. The following seems the programme they themselves would have most liked :I. Mathematics, rigorously taught up to their capacity, and copiously illustrated and applied, so as to throw as much interest into its pursuit as possible. 2. Logic. 3. Some branch of science (observation, theory, and experiment), some boys taking one branch and some another, to insure variety of interests under the same roof. 4 . Accurate drawing of objects connected with that branch of science. 5. Mechanical handiwork. All these to be rigorously taught. The following not to be taught rigorously : reading good books (not trashy ones) in literature, history, and art. A moderate knowledge of the more useful languages taught in the easiest way, probably by going abroad in vacations. It is abundantly evident that the leading men of science have not been made by much or regular teaching. They craved for variety. Those who had it, praised it ; and those who had it not, concurred in regretting it. There were none who had the oldfashioned high-and-dry education who were satisfied with it. Those who came from the greater schools usually did nothing there, and have abused the system heartily.
INFLUENCE OF GEOLOGICAL CHANGES ON THE EARTH'S ROTATION

$A \mathrm{~T}$ the annual meeting of the Geological Society of A Glasgow, on Feb. I2, the president, Sir William Thomson, F.R.S., gave an address on the above subject, of which the following is an abstract:-

$\mathrm{He}$ first briefly considered the rotation of rigid bodies in general, defining a principal axis of rotation as one for which the centrifugal forces balance while the body $\mathrm{rcm}$ tates around it. He then took the case of the earth; and, having pointed out the position of its present axis, showed that if from any cause it were made to revolve round any other, that would be an "instantaneous axis," changing every instant, and travelling through the solid, from west to east, in a period of 296 days round the principal axis It would shift continually in the figure, owing to the varying centrifugal force of two opposite portions of the body. This would produce, by centrifugal force, a tide of peculiar distribution over the ocean, having 296 days for period. An inclination of the axis of instantaneous rotation to principal axis of $\mathrm{I}^{\prime \prime}$, or $100 \mathrm{ft}$. at the earth's surface, would produce rise and fall of water in $45^{\circ}$ latitude, where the effect is greatest, amounting to 'I 7 of a foot above and below mean level.

$\mathrm{He}$ noticed, in passing, the application of these dynamical principles to the attraction which the sun and moon exercise on the protuberant parts of the earth, tending to bring the plane of the earth's equator into coincidence with the ecliptic. This causes an incessant change, to a certain limited extent, in the position of the axis of rotation, thereby occasioning what is known as the "precession of the equinoxes." Having illustrated these remarks by some interesting experiments, Sir William Thomson proceeded to consider more particularly the circumstances according to which the axis of the earth might become changed through geological infuences, and the consequences of any such change. The possibility of such a change had been adduced to account for the great differences in climate which can be shown to have obtained at different periods in the same portion of the earth's surface. In the British Isles, for example, and in many other countries, there is clear evidence that at a comparatively recent period a very cold climate-much colder than at present-prevailed; while in the same places the remains of plants and animals belonging to several preceding eras indicate a high temperature and a comparatively tropical climate. The question arose, can changes in the earth's axis account for these changes of climate? In the present condition of the earth, any change in the axis of rotation could not be permanent, because the instantaneous axis would travel round the principal axis of the solid in a period of 296 days, as already stated. Maxwell had pointed out that this shifting of the instantaneous axis in the solid would constitute in its period a periodic variation everywhere of "latitude," ranging above and below the mean value, to an extent equal to the angular deviation of the instantaneous axis of rotation from the principal axis; and, by comparing observations of the altitude of the Pole-star during three years at Greenwich, had concluded that there may possibly be as much as $\frac{7}{2}$ " of such deviation, but not more.

In very early geologic ages, if we suppose the earth to have been plastic, the yielding of the surface might have made the new axis a principal axis. But certain it is that the earth at present is so rigid that no such change is possible. The precession of the equinoxes shows that the earth at present moves as a rigid body; and during the whole period of geologic history, or while it has been inhabited by plants and animals, it has been practically rigicl. Changes of climate, then, have not been produced by changes of the axis of the earth. The learned professor then inquired what influences great subs: 'ences or 